\title{
Early Hematopoietic Stem Cell Transplant is Associated with Favorable Outcomes in Children with MDS
}

\author{
Angela R. Smith, M.D., M.S. ${ }^{1}$, Ellen C. Christiansen, B.A. ${ }^{2}$, John E. Wagner, M.D. ${ }^{1}$, Qing \\ Cao, PhD ${ }^{3}$, Margaret L. MacMillan, M.D. ${ }^{1}$, Heather E. Stefanski, M.D., PhD ${ }^{1}$, Barbara A. \\ Trotz, B.S. ${ }^{4}$, Michael J. Burke, M.D. ${ }^{4}$, and Michael R. Verneris, M.D. ${ }^{1}$ \\ ${ }^{1}$ Division of Pediatric Blood and Marrow Transplantation, University of Minnesota \\ 2University of Minnesota Medical School \\ ${ }^{3}$ Masonic Cancer Center Biostatistics Core, University of Minnesota \\ ${ }^{4}$ Division of Pediatric Hematology/Oncology, University of Minnesota
}

\begin{abstract}
Background-Although hematopoietic stem cell transplantation (HSCT) is the treatment of choice for childhood myelodysplastic syndrome (MDS), there is no consensus regarding patient or disease characteristics that predict outcomes.
\end{abstract}

Procedure-We reviewed 37 consecutive pediatric MDS patients who received myeloablative HSCT between 1990 and 2010 at a single center.

Results-Twenty had primary MDS and 17 had secondary MDS. Diagnostic cytogenetics included monosomy $7(n=21)$, trisomy $8(n=7)$ or normal/other $(n=8)$. According to the modified WHO MDS classification, thirty had refractory cytopenia and 7 had refractory anemia with excess blasts. IPSS scores were: low risk $(n=1)$, intermediate-1 $(n=15)$, and intermediate-2 $(n=21)$. OS and DFS at 10-years in the entire cohort was 53\% and 45\%. Relapse at 10-years was $26 \%$ and 1year TRM was $25 \%$. In multivariate analysis, factors associated with improved 3-year DFS were not receiving pre-HSCT chemotherapy $(\mathrm{RR}=0.30,95 \% \mathrm{CI} 0.10-0.88 ; \mathrm{p}=0.03)$ and a shorter interval ( $<140$ days) from time of diagnosis to transplant $(R R=0.27$, 95\% CI 0.09-0.80; $p=0.02)$. 3-year DFS in patients who did not receive pre-HSCT chemotherapy and those who had a shorter interval to transplant $(\mathrm{n}=16)$ was $80 \%$.

Conclusion-These results suggest that children with MDS should be referred for allogeneic HSCT soon after diagnosis and that pre-HSCT chemotherapy does not appear to improve outcomes.

\section{INTRODUCTION}

\begin{abstract}
Myelodysplastic syndrome (MDS) is a clonal disorder of hematopoiesis with variable bone marrow dysplasia and cellularity, progressive cytopenias, and a propensity for transformation to acute myelogenous leukemia (AML) [1]. It is heterogeneous in presentation and rare in children, with distinct features in pediatric patients as compared to adults. These features have impeded the diagnosis, classification, and scientific understanding of this disease in the past [2]. Recently strides have been made toward the classification of pediatric MDS, with new acceptance of minimal diagnostic criteria. Though
\end{abstract}

Corresponding Author: Angela R. Smith, M.D., M.S. 420 Delaware Street SE; MMC 484 Minneapolis, MN 55455, USA Phone: 612-626-2778 Fax: 612-626-2815 smith719@umn.edu.

FINANCIAL DISCLOSURES: The authors have no relevant conflicts of interest to disclose. 
a blurring of clinical categories may still exist, pediatric MDS is understood to be a distinct disease from myeloid leukemia of Down syndrome and juvenile myelomonocytic leukemia (JMML), and is composed of the following subdivisions: refractory cytopenia (RC), refractory anemia with excess blasts (RAEB) and RAEB in transformation (RAEB-T) [3].

Hematopoietic stem cell transplant (HSCT) is the only curative therapy for pediatric MDS, although other therapies have been attempted including immunosuppressive therapy, epigenetic modifying agents, high-dose chemotherapy, and hematopoietic growth factors [47]. With continued refinements in HLA-typing, availability of umbilical cord blood and improvements in supportive care management, the availability of HSCT has increased and outcomes have improved. Today, outcomes for unrelated donor HSCT for hematologic diseases are similar to those seen in recipients of HLA matched related donor (MRD) [716]. Although an improvement in survival has been reported, treatment related mortality (TRM) and relapse remain the most common causes of death $[7,9,12,15,17]$.

There has been little consensus regarding patient or disease characteristics, or treatmentrelated factors that may be associated with transplant outcomes for pediatric MDS. This is likely related to small patient samples and challenges in diagnosis and classification of MDS in children. Previously reported prognostic factors have included donor type, French American British (FAB) subtype, bone marrow and peripheral blood blast percentage, time from diagnosis to HSCT, age, cytopenias, trephine biopsy characteristics, and karyotype [12-16,18-20]. The international prognostic scoring system (IPSS), which has effectively correlated disease factors at presentation to outcomes in adults, has been applied to the pediatric population; however, results are of limited value as only BM blasts $<5 \%$ and platelet count $>100 \times 10^{9} / \mathrm{L}$ were found to successfully predict survival [21]. Worse outcomes have also been observed in patients with secondary MDS, defined as MDS arising either after prior chemotherapy, aplastic anemia or a bone marrow failure syndrome, or familial MDS [17,22].

Because MDS is a rare pediatric disease, the majority of the literature on outcomes and prognostic factors are from registry studies. While of clear value, such studies are limited by heterogeneous treatment and supportive care regimens. In this study we report the results for 37 consecutive pediatric patients with MDS undergoing HSCT at the University of Minnesota to determine whether patient, disease or treatment characteristics can be identified predicting post-HSCT outcomes.

\section{MATERIALS AND METHODS}

\section{Study design}

We performed a retrospective review of 37 consecutive pediatric patients $(<21$ years old at diagnosis) who received allogeneic HSCT for MDS between August 1990 and May 2010. All were transplanted on institutional review board approved treatment protocols, and all patients/guardians provided signed informed consent.

\section{Donor selection and HLA typing}

Stem cell sources included HLA MRD bone marrow, matched or mismatched unrelated donor (MURD or MMURD) bone marrow, and unrelated umbilical cord blood (UCB). For recipients of UCB, patients and donors were typed for HLA-A and -B at the antigen level and for-DRB1 at the allele level. For recipients of MURD and MMURD, patients and donors had antigen level typing at HLA-A and -B and allele level typing at HLA-DRB1 until June 2005, when testing at the allele level for all loci, including C, was implemented. All HLA matched related donors had antigen level typing at HLA-A, -B, and -DRB1 unless 
adequate family typing was not available to determine haplotypes, in which case allele level DNA typing was performed at all loci when available after 2005.

\section{Supportive care}

Patients were hospitalized in single rooms ventilated with high-efficiency particulate air filtration systems. Patients at high risk for recurrence of herpes simplex (titer $\geq 1: 8$ ) received prophylactic acyclovir. Patients at high risk of cytomegalovirus (CMV) reactivation (recipient or donor with a titer $\geq 1: 8$ ) received prophylactic high-dose acyclovir until day 100. Broad-spectrum antibiotics were administered for fever during aplasia, and antifungals were added for persistent fever unresponsive to antibiotic therapy. All patients received fluconazole for prophylaxis of yeast infections for 100 days, trimethoprim-sulfamethoxazole for prophylaxis of Pneumocystis (carinii) jiroveci after engraftment for 12 months after transplantation, and antibiotic prophylaxis (penicillin, gatifloxacin, or levofloxacin) for Gram-positive organisms during the treatment of GVHD.

\section{Endpoints}

Study endpoints included neutrophil engraftment, overall survival (OS), disease free survival (DFS), relapse, acute graft-versus-host-disease (aGVHD; grades II-IV and grades III-IV), chronic GVHD (cGVHD), and treatment-related mortality (TRM). Neutrophil engraftment was defined as the first of 3 consecutive days with an absolute neutrophil count (ANC) $>0.5 \times 10^{9} / \mathrm{L}$. OS was defined as the time from transplant (day 0 ) until death or last contact. DFS was defined as the time from transplant (day 0) until disease recurrence, death, or last patient contact, whichever came first. Relapse was defined as a morphologic and/or cytogenetic recurrence of MDS after HSCT. Acute GVHD and cGVHD were evaluated and graded according to previously published criteria, with histopathologic confirmation when possible $[23,24]$. All patients with engraftment were considered evaluable for GVHD. TRM was defined as death in the first 100 days after HSCT for any reason other than relapse.

\section{Statistical Methods}

Data on transplant patient characteristics, post-transplantation complications and outcomes were collected prospectively by the Biostatistical Support Group at the University of Minnesota using standardized collection procedures. Patients and disease characteristics were summarized using descriptive statistics. All patients were followed longitudinally until death or last follow up. Kaplan-Meier was used to estimate neutrophil recovery, OS and DFS [25]. Cumulative incidence was used to estimate relapse, TRM, aGVHD, and cGVHD. Statistical comparison of neutrophil engraftment, OS and DFS between groups was completed by the Log-Rank test [26]. The proportional hazards model of Fine and Gray was used to assess the independent factors on relapse, TRM, aGVHD, and cGVHD [27]. Statistical analysis was performed with Statistical Analysis System statistical software version 9.2 (SAS Institute). Groups with value of $p \leq 0.05$ were considered to be statistically different.

\section{RESULTS}

\section{Patient and Disease Characteristics}

Patient characteristics are summarized in Table I. Twenty patients (54\%) had primary (de novo) MDS and 17 (46\%) had secondary MDS (4 treatment-related, 8 with preceding idiopathic aplastic anemia, 3 with Schwachman-Diamond syndrome, and 2 familial [defined as MDS occurring in one or more first degree relatives]). Those with juvenile myelomonocytic leukemia, Fanconi anemia, myelodyplasia-related AML and MDS associated with Down Syndrome were excluded. Patients were retrospectively assigned a 
modified World Health Organization (WHO) classification and scored according to the International Prognostic Scoring System (IPSS) [3][28]. Thirty patients (81\%) had refractory cytopenia (RC) and 7 (19\%) had refractory anemia with excess blasts (RAEB) according to the modified WHO MDS classification. The IPSS risk of our patients was as follows: low $(\mathrm{n}=1)$, intermediate-1 (Int-1; $\mathrm{n}=15)$, intermediate-2 $(\mathrm{Int}-2 ; \mathrm{n}=21)$, high $(\mathrm{n}=0)$. Seven patients (19\%) received pre-HSCT chemotherapy which comprised of AML induction chemotherapy (DCTER regimen $\mathrm{n}=3$, COG AAML0531 n=1, Ara-c/mitoxantrone + FLAG/Ida n=1, unknown $\mathrm{n}=1$ ) with the exception of one patient who received only cis-retinoic acid. No patients received DNA methyltransferase inhibitor (DNAMTi) agents. The decision to use pre-HSCT chemotherapy was at the discretion of the treating physician. Response to the preHSCT chemotherapy was mixed -3 had worsening blasts counts, 2 had stable blast counts and 2 had improved blast counts. There was no correlation between blast count at diagnosis or interval from diagnosis to HSCT and the use of pre-HSCT chemotherapy. All patients were transplanted following myeloablative conditioning (cyclophosphamide/total body irradiation [ $\mathrm{n}=29$ ], cyclophosphamide/fludarabine/total body irradiation [ $\mathrm{n}=6]$, busulfan/ cyclophosphamide $[\mathrm{n}=1]$ and busulfan/fludarabine/melphalan $[\mathrm{n}=1])$. Donor sources included UCB (HLA matched [6/6], $n=1$; HLA mismatched at 1 or 2 loci [4/6 or 5/6], n=8), $\operatorname{MRD}(n=15), \operatorname{MURD}(n=7)$ and MMURD [5/6] $(n=6)$. Median length of follow-up for survivors was 8 years (range 1-17).

\section{Hematopoietic Recovery and Engraftment}

Neutrophil engraftment occurred in 89\% (95\% CI 77-97\%) of patients at a median of 23 days (range, 12-40). Multivariate analysis demonstrated that patients transplanted after 1999 were more likely to engraft by day +42 (RR $2.27,95 \%$ CI $1.06-4.88 ; \mathrm{p}=0.04)$. There were no other significant predictors of engraftment.

\section{Overall Survival}

Overall survival was 70\% (95\% CI 53-82\%) and 53\% (95\% CI 36-68\%) at 1 and 3-years. Survival remains $53 \%$ at 10 years. (Figure 1A) Univariate analysis revealed improved 3-year OS in patients transplanted after 1999 (85\% vs. 38\%, p=0.02), those who had a pre-HSCT blast count of $<5 \%$ (66\% vs. $20 \%$, $\mathrm{p}=0.01$ ), those who had an MDS classification of RC ( $61 \%$ vs. $17 \%, \mathrm{p}=0.03$ ), those who did not receive pre-HSCT chemotherapy (63\% vs. $14 \%$, $\mathrm{p}<0.01)$ and those who had a shorter interval from time of diagnosis to HSCT ( $<140$ days) ( $71 \%$ vs. $36 \%, \mathrm{p}=0.02$ ). In multivariate analysis (Table II), 3-year OS was greater in patients who did not receive pre-HSCT chemotherapy (RR of death $0.29,95 \%$ CI $0.04-0.78, p=0.02$ ) and in those who received HSCT after 1999 (RR 0.17, 95\% CI 0.04-0.78; $\mathrm{p}=0.02$ ). MDS group, cytogenetics, IPSS risk, time from diagnosis to transplant, pre-HSCT BM blast percentage and donor source had no impact on 3-year OS in multivariate analysis.

\section{Disease Free Survival}

DFS was 62\% (95\% CI 44-75\%) and 48\% (95\% CI 31-63\%) at 1 and 3-years. At 10 years DFS is similar at $45 \%$ (95\% CI 28-60\%) (Figure 1B) In univariate analysis, improved DFS at 3-years was found for patients who did not receive pre-HSCT chemotherapy (60\% vs. $0 \%, \mathrm{p}<0.01$ ) (Figure 2A), those who had a pre-HSCT BM blast percentage $<5 \%$ (59\% vs. $20 \%, \mathrm{p}=0.04)$ and those who had a time from diagnosis to HSCT of $<140$ days $(71 \%$ vs. $26 \%, \mathrm{p}<0.01$ ) (Figure 2B). Decreased DFS was observed in recipients of MMURD marrow ( $17 \%$ vs. $65 \%$ in UCB, $53 \%$ in MRD and $43 \%$ in MURD, $p=0.03$ ). In multivariate analysis (Table II) improved DFS at 3-years was associated with not receiving pre-HSCT chemotherapy (RR 0.30, 95\% CI 0.10-0.88; $\mathrm{p}=0.03$ ) and a shorter interval ( $<140$ days) from time of diagnosis to HSCT (RR $0.27,95 \%$ CI 0.09-0.80; $\mathrm{p}=0.02$ ). Patients with an IPSS risk of Int- 2 had a trend toward lower DFS although this was not quite statistically significant (RR 2.97, 95\% CI 0.95-9.33; p=0.06). Cytogenetics, pre-HSCT BM blast percentage and 
donor source had no significant impact on 3-year DFS in multivariate analysis. Because preHSCT chemotherapy and interval from diagnosis to transplant were clearly associated with outcome, we also analyzed this group separately. There were 16 patients who did not receive pre-HSCT chemotherapy and were transplanted $<140$ days from diagnosis. Donor sources for this group included 5 UCB, 3 MURD and 8 MRD. Three year OS and DFS were both $80 \%$ (95\% CI 51-93\%) (Figure 2C).

\section{Relapse}

The incidence of relapse at 2-years was 20\% (95\% CI 7-33\%). Factors associated with decreased risk of relapse at 2-years in multivariate analysis were secondary MDS (RR 0.12, 95\% CI 0.02-0.86; $\mathrm{p}=0.03$ ) and not receiving pre-HSCT chemotherapy (RR 0.13, 95\% CI $0.03-0.52 ; \mathrm{p}<0.01)$. A shorter time from diagnosis of MDS to HSCT was associated with lower relapse in univariate analysis ( $11 \%$ vs. $28 \%, \mathrm{p}=0.05)$, but this association did not hold true in multivariate analysis. Pre-HSCT BM blast percentage did not significantly affect relapse rates in either the univariate analysis or multivariate analysis. Additionally, the presence of aGVHD and cGVHD was not correlated with relapse.

\section{Treatment Related Mortality and GVHD}

Treatment-related mortality was $25 \%$ (95\% CI 11-39\%) at 2-years. In multivariate analysis, the risk of TRM was increased in those who had a time from diagnosis to HSCT of $\geq 140$ days (RR 9.86, 95\% CI 1.28-76.22; p=0.03). MDS group, IPSS risk, pre-HSCT blast percentage, pre-HSCT chemotherapy, transplant year and donor source had no significant impact on TRM. The incidence of grade II-IV and III-IV aGVHD at 100 days was $41 \%$ (95\% CI 24-57\%) and 16\% (95\% CI 5-28\%), respectively, while the 1-year incidence of cGVHD was $19 \%$ (95\% CI 6-32\%).

\section{DISCUSSION}

In this single center study, we show that pediatric patients with MDS who received myeloablative HSCT early after the diagnosis and without pre-HSCT chemotherapy showed significantly improved outcomes (3 year OS and DFS of $80 \%$ ) relative to those transplanted $>140$ days from diagnosis and/or after induction chemotherapy. The combination of these two variables and their association with improved outcomes is novel and has not been previously reported. While they need to be validated, these results suggest that proceeding to HSCT early in the disease is indicated.

Survival rates in this series are similar to previously published results [12,15]. Our patients had an estimated OS of $53 \%$ and DFS of $48 \%$ at 3-years. Remarkably, these survival rates are essentially unchanged at 10 years post HSCT. Published data are limited, as many studies define MDS with different parameters, with some including the diagnoses CMML, JMML, and MDS-AML. Among studies limiting MDS to include RA/RARS (refractory anemia with ringed sideroblasts), RAEB, and RAEB-T according to the FAB or modified WHO classification, estimates of disease- or event-free survival range anywhere from 38 to $60.9 \%$ at 10 months to 8 years $[8,12-14,16]$. In our study, OS, DFS, and engraftment results improved after 1999. Advances in supportive care and HLA-typing likely are major contributors to this improvement, as has been previously demonstrated in adults [29]. Woodard et al., in their evaluation of unrelated HSCT for MDS, also reported decreased cGVHD after 1994, which the authors attribute to improved HLA-matching [12]. Rocha et al. similarly reported year of transplant as a predictive factor, but cite decreased TRM after 2001 as the reason [13]. 
In our study, TRM was the greatest contributor to mortality at $25 \%$. Several other pediatric studies report similar TRM rates, with a range of 21-59\% [9,12,14,15,17,30]. Because of this, reduced intensity conditioning (RIC) HSCT has been investigated and shown to be well tolerated among adults with hematologic malignancies [31]. Although these studies show decreased TRM, they report higher relapse rates, leading to an overall similar DFS [32-34]. RIC HSCT outcomes in children are more limited, as this conditioning approach is less prevalent in pediatrics. Strahm et al., in a prospective pilot study of the European Working Group for MDS, reported an EFS of 74\% after RIC HSCT, which is similar to survival rates following standard myeloablative conditioning with HLA-identical donors [9,35]. Andolina et al., however, reported a lower relapse free survival amongst children receiving RIC HSCT for MDS (47\%) [36]. Further pediatric prospective studies will be necessary to delineate whether RIC can effectively reduce TRM while improving overall survival rates in MDS.

We identified improved DFS in patients who had a shorter time from diagnosis to HSCT (defined as $<140$ days). These results are consistent with those published by Rocha et al. where among pediatric patients receiving unrelated UCB transplant $<6$ months after diagnosis, there was a $66 \% 3$-year probability of DFS, compared to $20 \%$ if HSCT was delayed [13]. Strahm et al. reported mixed results for early transplant ( $<4$ months from diagnosis to HSCT) in advanced MDS, citing reduced TRM, but increased relapse [15]. In contrast, our analysis identified no correlation between the time from diagnosis to HSCT and relapse. The exact explanation of why early transplantation might be associated with improved outcomes is not entirely clear, but may be due to less transfusion support and lower rates of allo-sensitization. As the number of studies commenting on the importance in the time from diagnosis to transplant is limited, further comparison is difficult. Our results suggest that early referral for HSCT after diagnosis is preferred when possible.

Patients in our study who did not receive pre-HSCT chemotherapy had better outcomes (improved OS, DFS and relapse rates) than those who received chemotherapy. In adults, pre-HSCT chemotherapy resulting in a complete remission (BM blast count $<5 \%$ ) marrow is associated with lower relapse [37]. In our study, however, a BM blast count $<5 \%$ did not significantly affect relapse rates nor was it associated with pre-HSCT chemotherapy use. Historically, pediatric patients have been treated similarly to adults with MDS, and children have tolerated intensive chemotherapy prior to HSCT well with limited toxicities [38]. However, pediatric MDS studies report a relatively poor response to chemotherapy prior to HSCT $[39,40]$, and intensive chemotherapy and lower blast counts prior to transplant are not associated with improved relapse rates, survival, or lower TRM [12,15]. As all of our patients received myeloablative conditioning, we cannot comment on blast count or preHSCT chemotherapy prior to a RIC HSCT; however, our results would suggest that chemotherapy before myeloablative HSCT for pediatric MDS is associated with worse outcomes.

As with any single institution report, the major limitation to this study is the small sample size which limits the applicability of the results without further confirmation in larger groups of patients. Additionally, given the retrospective nature of the report, the cohort was heterogeneous with regard to diagnostic methods, pre-HSCT chemotherapy, timing of referral and donor source which also limits the strength of the conclusions that can be drawn. However, all patients were treated at a single transplant institution and therefore transplant procedures, HLA typing, supportive care and post transplant care was uniform.

In summary, this study demonstrates the importance of early HSCT without prior chemotherapy in children with MDS. Some caution must be invoked when interpreting our results, however, considering that the approach to MDS has evolved with the FDA approval of DNA methyltransferase inhibitors like 5-azacytidine and decitabine. While this class of 
drugs is not curative, they do have the potential to modify the course of MDS, perhaps altering the burden of malignant cells. As well, they have the potential to alter sensitivity to chemotherapy and/or alloreactive (graft-versus-leukemia) responses [41]. Data on the effect of pre-HSCT hypomethylation on post-HSCT outcomes are limited. Retrospective reports in adults show that the treatment is well tolerated and that outcomes are at least similar to historical controls receiving induction chemotherapy or no pre-HSCT therapy $[42,44]$. The effect of these agents on pediatric MDS is remains unknown, highlighting the need for cooperative group national and/or international trials. We look forward to future studies examining whether use hypomethylating agents, either before or after transplantation, might impact overall outcomes. Future studies focusing on the use of RIC regimens with the goal of reducing TRM and late effects in long term survivors are also needed.

\section{REFERENCES}

1. Smith, FO.; Woods, WG. Myeloproliferative and myelodysplastic disorders. In: Pizzo, PA.; Poplack, DG., editors. Principles and practice of pediatric oncology. Lippincott Williams \& Wilkins; Philadelphia: 2002. p. 615

2. Niemeyer C, Baumann I. Myelodysplastic syndrome in children and adolescents. Semin.Hematol. 2008; 45:60-70. [PubMed: 18179970]

3. Hasle H, Niemeyer CM, Chessells JM, et al. A pediatric approach to the WHO classification of myelodysplastic and myeloproliferative diseases. Leukemia. 2003; 17:277-282. [PubMed: 12592323]

4. Hasle H. Myelodysplastic and myeloproliferative disorders in children. Curr Opin Pediatr. 2007; 19:1-8. [PubMed: 17224655]

5. Locatelli F, Zecca M, Pession A, Maserati E, De Stefano P, Severi F. Myelodysplastic syndromes: the pediatric point of view. Haematologica. 1995; 80:268-279. [PubMed: 7672722]

6. Hasegawa D, Manabe A, Yagasaki H, et al. Treatment of children with refractory anemia: the Japanese Childhood MDS Study Group trial (MDS99). Pediatr Blood Cancer. 2009; 53:1011-1015. [PubMed: 19499580]

7. Stary J, Locatelli F, Niemeyer CM. Stem cell transplantation for aplastic anemia and myelodysplastic syndrome. Bone Marrow Transplant. 2005; 35:S13-S16. [PubMed: 15812522]

8. Guinan EC, Tarbell NJ, Tantravahi R, Weinstein HJ. Bone marrow transplantation for children with myelodysplastic syndromes. Blood. 1989; 73:619-622. [PubMed: 2644982]

9. Yusuf U, Frangoul HA, Gooley TA, et al. Allogenic bone marrow transplantation in children with myelodysplastic syndrome or juvenile myelomonocytic leukemia: the Seattle experience. Bone Marrow Transplant. 2004; 33:805-814. [PubMed: 14755311]

10. Trobaugh Lotrario AD, Kletzel M, Quinones RR, et al. Monosomy 7 associated with pediatric acute myeloid leukemia (AML) and myelodysplastic syndrome (MDS): successful management by allogeneic hematopoietic stem cell transplant (HSCT). Bone Marrow Transplant. 2005; 35:143149. [PubMed: 15558042]

11. Davies SM, Wagner JE, Defor T, et al. Unrelated donor bone marrow transplantation for children and adolescents with aplastic anaemia or myelodysplasia. Br.J.Haematol. 1997; 96:749-756. [PubMed: 9074418]

12. Woodard P, Carpenter P, Davies S, et al. Unrelated donor bone marrow transplantation for myelodysplastic syndrome in children. Biol Blood Marrow Transplant. 2011; 17:723-728. [PubMed: 20813197]

13. Rocha V, Kabbara N, Ionescu I, Ruggeri A, Purtill D, Gluckman E. Pediatric related and unrelated cord blood transplantation for malignant diseases. Bone Marrow Transplant. 2009; 44:653-659. [PubMed: 19802021]

14. Parikh S, Mendizabal A, Martin P, et al. Unrelated donor umbilical cord blood transplantation in pediatric myelodysplastic syndrome: a single-center experience. Biol Blood Marrow Transplant. 2009; 15:948-955. [PubMed: 19589484] 
15. Strahm B, Nllke P, Zecca M, et al. Hematopoietic stem cell transplantation for advanced myelodysplastic syndrome in children: results of the EWOG-MDS 98 study. Leukemia. 2011; 25:455-462. [PubMed: 21212791]

16. Muoz A, Daz-Heredia C, Badell I, et al. Allogeneic stem cell transplantation for myelodysplastic syndromes in children: a report from the Spanish Working Party for Blood and Marrow Transplantation in Children (GETMON). Pediatr HematolOncol. 2009; 26:345-355.

17. Woodard P, Barfield R, Hale G, et al. Outcome of hematopoietic stem cell transplantation for pediatric patients with therapy-related acute myeloid leukemia or myelodysplastic syndrome. Pediatr Blood Cancer. 2006; 47:931-935. [PubMed: 16155933]

18. Tricot G, Vlietinck R, Boogaerts MA, et al. Prognostic factors in the myelodysplastic syndromes: importance of initial data on peripheral blood counts, bone marrow cytology, trephine biopsy and chromosomal analysis. Br J Haematol. 1985; 60:19-32. [PubMed: 3859319]

19. Mangi MH, Mufti GJ. Primary myelodysplastic syndromes: diagnostic and prognostic significance of immunohistochemical assessment of bone marrow biopsies. Blood. 1992; 79:198-205. [PubMed: 1370203]

20. Mufti GJ, Stevens JR, Oscier DG, Hamblin TJ, Machin D. Myelodysplastic syndromes: a scoring system with prognostic significance. Br J Haematol. 1985; 59:425-433. [PubMed: 3970861]

21. Hasle H, Baumann I, Bergstrsser E, et al. The International Prognostic Scoring System (IPSS) for childhood myelodysplastic syndrome (MDS) and juvenile myelomonocytic leukemia (JMML). Leukemia. 2004; 18:2008-2014. [PubMed: 15496981]

22. Sasaki H, Manabe A, Kojima S, et al. Myelodysplastic syndrome in childhood: a retrospective study of 189 patients in Japan. Leukemia. 2001; 15:1713-1720. [PubMed: 11681412]

23. MacMillan M, Weisdorf D, Wagner J, et al. Response of 443 patients to steroids as primary therapy for acute graft-versus-host disease: comparison of grading systems. Biol Blood Marrow Transplant. 2002; 8:387-394. [PubMed: 12171485]

24. Horwitz M, Sullivan K. Chronic graft-versus-host disease. Blood Rev. 2006; 20:15-27. [PubMed: 16426941]

25. Kaplan E, Meier P. Nonparametric estimation from incomplete observations. J Am Stat Assoc. 1958; 53:457-481.

26. Lin D. Non-parametric inference for cumulative incidence functions in competing risks studies. Stat Med. 1997; 16:901-910. [PubMed: 9160487]

27. Fine J, Gray R. A proportional hazards model for the subdistribution of a competing risk. J Am Stat Assoc. 1999:496-509.

28. Greenberg P, Cox C, LeBeau MM, et al. International scoring system for evaluating prognosis in myelodysplastic syndromes. Blood. 1997; 89:2079-2088. [PubMed: 9058730]

29. Gooley T, Chien J, Pergam S, et al. Reduced mortality after allogeneic hematopoietic-cell transplantation. N Eng.J Med. 2010; 363:2091-2101.

30. Kardos G, Baumann I, Passmore SJ, et al. Refractory anemia in childhood: a retrospective analysis of 67 patients with particular reference to monosomy 7. Blood. 2003; 102:1997-2003. [PubMed: 12763938]

31. Warlick E, Tomblyn M, Cao Q, et al. Reduced-intensity conditioning followed by related allografts in hematologic malignancies: long-term outcomes most successful in indolent and aggressive nonHodgkin lymphomas. Biol Blood Marrow Transplant. 2011; 17:1025-1032. [PubMed: 21047561]

32. Laport G, Sandmaier B, Storer B, et al. Reduced-intensity conditioning followed by allogeneic hematopoietic cell transplantation for adult patients with myelodysplastic syndrome and myeloproliferative disorders. Biol Blood Marrow Transplant. 2008; 14:246-255. [PubMed: 18215785]

33. Alyea E, Kim H, Ho V, et al. Comparative outcome of nonmyeloablative and myeloablative allogeneic hematopoietic cell transplantation for patients older than 50 years of age. Blood. 2005; 105:1810-1814. [PubMed: 15459007]

34. Martino R, Iacobelli S, Brand R, et al. Retrospective comparison of reduced-intensity conditioning and conventional high-dose conditioning for allogeneic hematopoietic stem cell transplantation using HLA-identical sibling donors in myelodysplastic syndromes. Blood. 2006; 108:836-846. [PubMed: 16597592] 
35. Strahm B, Locatelli F, Bader P, et al. Reduced intensity conditioning in unrelated donor transplantation for refractory cytopenia in childhood. Bone Marrow Transplant. 2007; 40:329-333. [PubMed: 17589538]

36. Andolina J, Kletzel M, Tse W, et al. Allogeneic hematopoetic stem cell transplantation in pediatric myelodysplastic syndromes: improved outcomes for de novo disease. Pediatr Transplant. 2011; 15:334-343. [PubMed: 21492354]

37. Warlick E, Cioc A, Defor T, Dolan M, Weisdorf D. Allogeneic stem cell transplantation for adults with myelodysplastic syndromes: importance of pretransplant disease burden. Biol Blood Marrow Transplant. 2009; 15:30-38. [PubMed: 19135940]

38. Woods WG, Kobrinsky N, Buckley J, et al. Intensively timed induction therapy followed by autologous or allogeneic bone marrow transplantation for children with acute myeloid leukemia or myelodysplastic syndrome: a Childrens Cancer Group pilot study. J Clin Oncol. 1993; 11:14481457. [PubMed: 8336184]

39. Woods W, Barnard D, Alonzo T, et al. Prospective study of 90 children requiring treatment for juvenile myelomonocytic leukemia or myelodysplastic syndrome: a report from the Children's Cancer Group. J Clin Oncol. 2002; 20:434-440. [PubMed: 11786571]

40. Hasle H, Kerndrup G, Yssing M, et al. Intensive chemotherapy in childhood myelodysplastic syndrome. A comparison with results in acute myeloid leukemia. Leukemia. 1996; 10:1269-1273. [PubMed: 8709630]

41. Choi J, Ritchey J, Prior JL, et al. In vivo administration of hypomethylating agents mitigate graftversus-host disease without sacrificing garft-versus-leukemia. Blood. 2010; 116(1):129-139. [PubMed: 20424188]

42. Gerds AT, Gooley TA, Estey EH. Pretransplantation therapy with azacytidine vs. induction chemotherapy and posttransplantation outcome in patients with MDS. Biol Blood Marrow Transplant. 2012; 18:1211-1218. [PubMed: 22252125]

43. Field T, Perkins J, Huang Y. 5-azacytidine for myelodysplasia before allogeneic hematopoietic cell transplantation. Bone Marrow Transplantation. 2010; 45:255-260. [PubMed: 19543327] 
A.

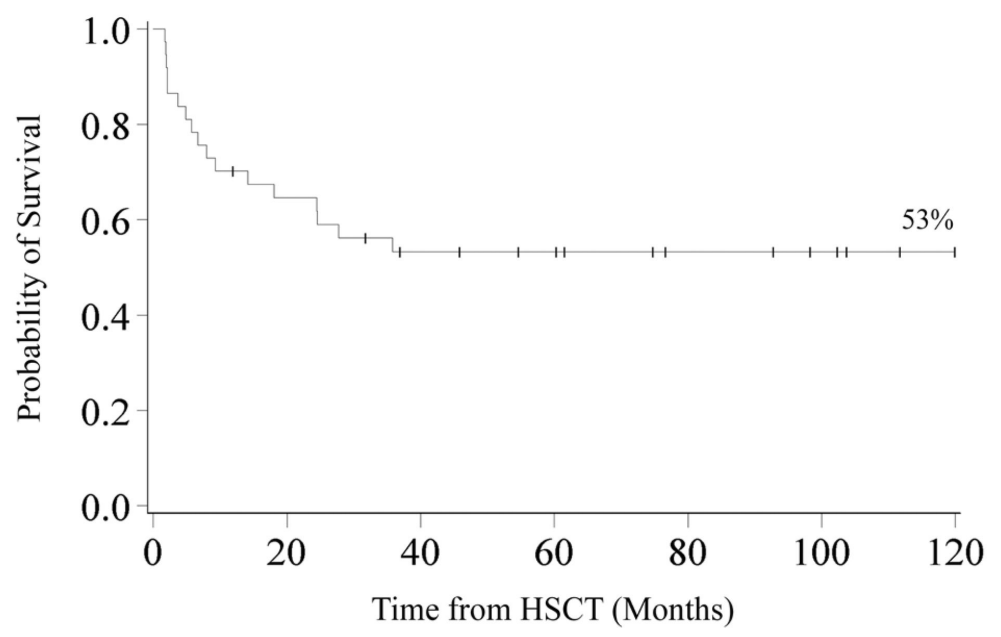

B.

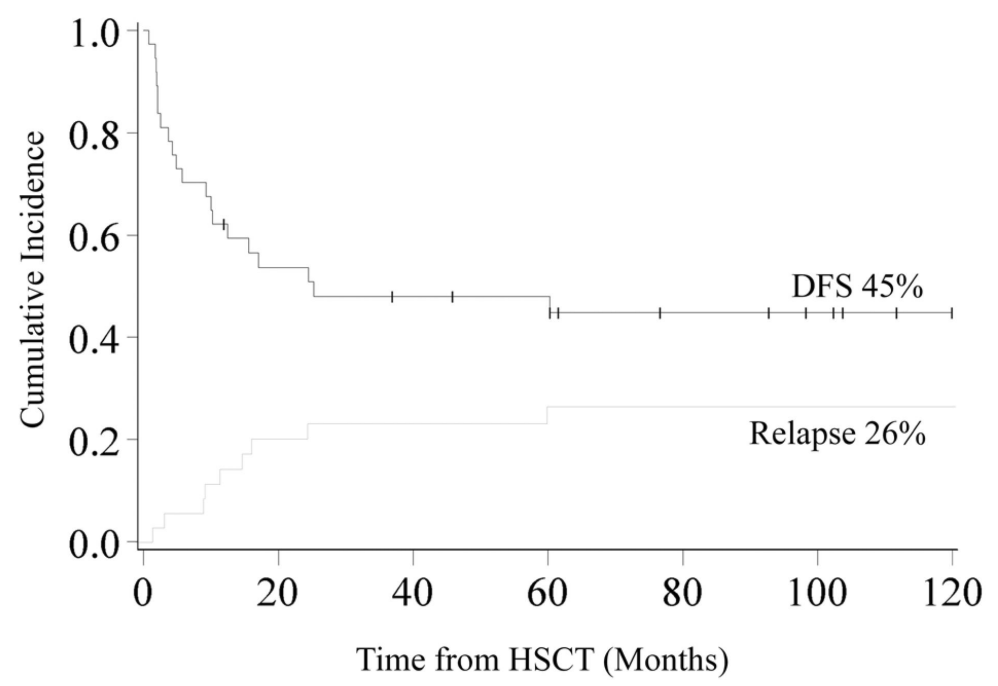

Figure 1. Kaplan-Meier Analysis of Overall Survival and Disease Free Survival A. Overall Survival. OS was 70\% (95\% CI 53-82\%) at 1 year and 53\% (95\% CI 36-68\%) at 3 years and beyond. B. Disease Free Survival and Relapse. DFS was $62 \%(95 \%$ CI $44-$ $75 \%)$ at 1 year and $48 \%(95 \%$ CI $31-63 \%)$ at 3 years and $45 \%(95 \%$ CI $28-60 \%)$ at 10 years. The incidence of relapse was $20 \%(95 \%$ CI $7-33 \%)$ at 2 years and $26 \%$ (95\% CI $11-$ $41 \%$ ) at 10 years. 
A.

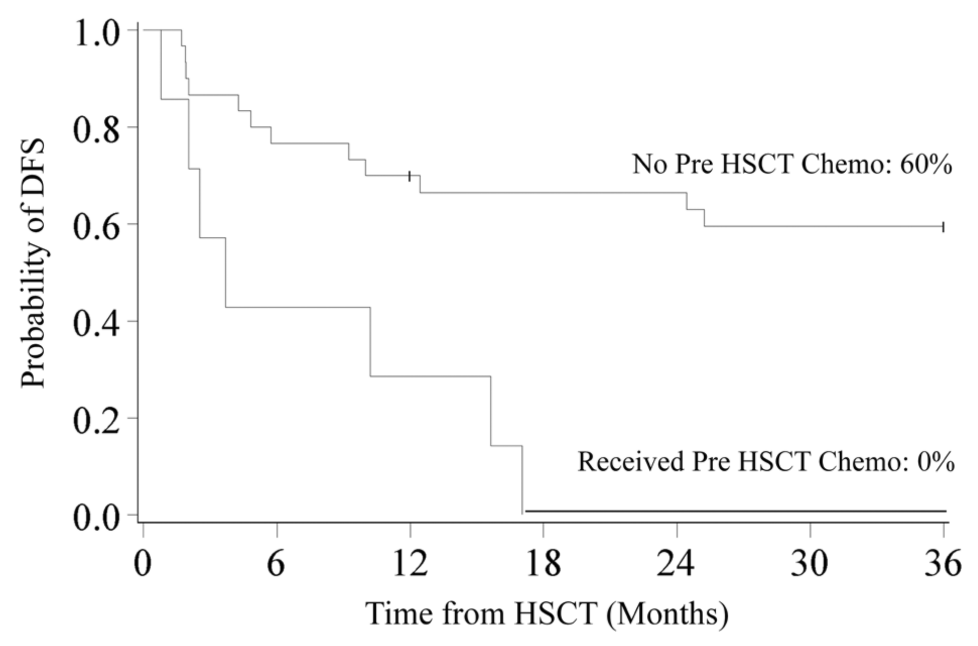

B.

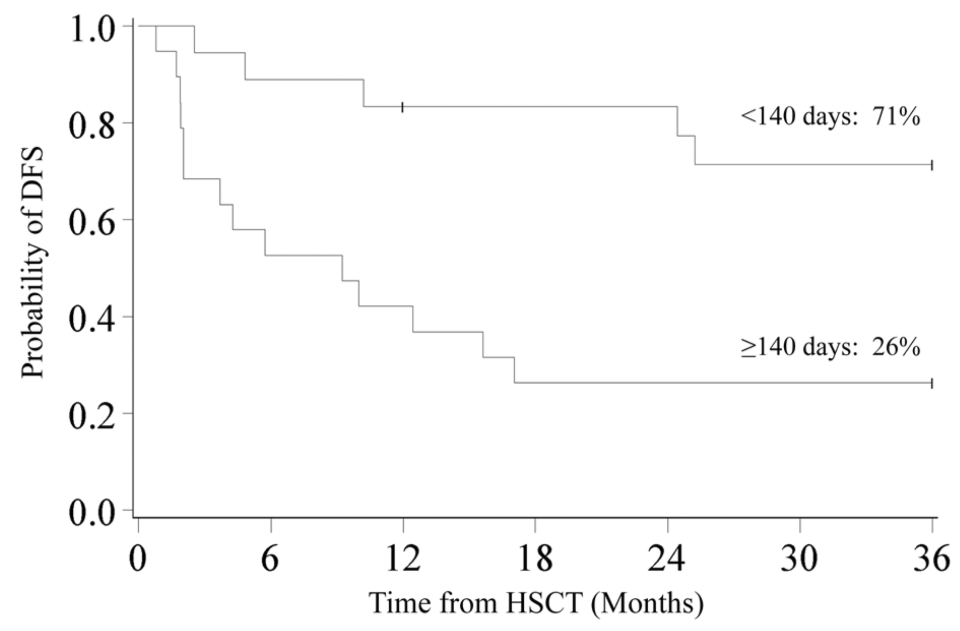

Pediatr Blood Cancer. Author manuscript; available in PMC 2014 April 01. 


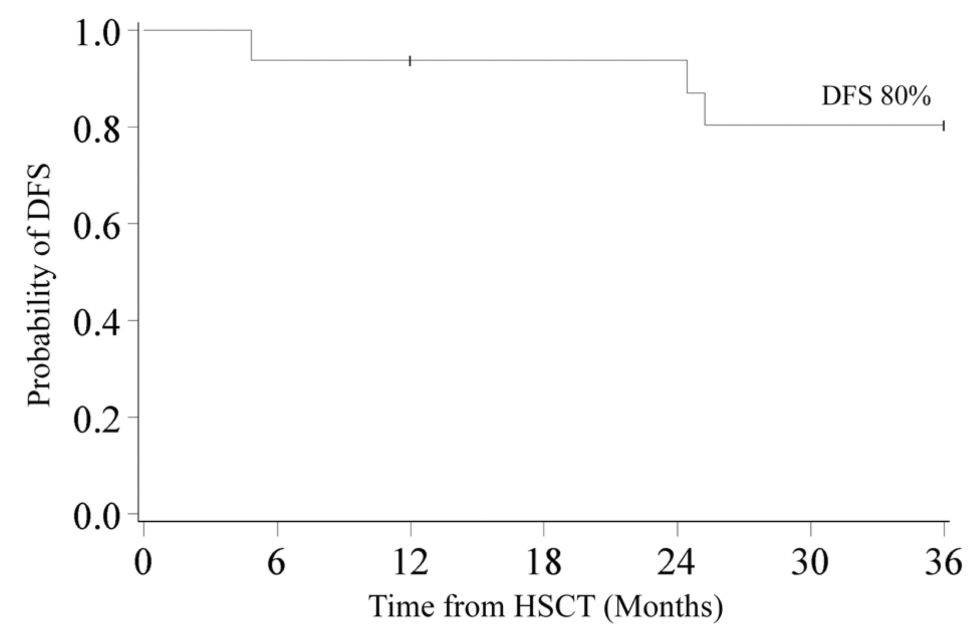

Figure 2.

A. Kaplan-Meier Analysis of 3-year DFS by Pre HSCT Chemotherapy Status. 3-year DFS was $60 \%$ (95\% CI 40-75\%) for patients who did not receive pre HSCT chemotherapy and $0 \%$ for patients who received pre HSCT chemotherapy $(\mathrm{p}<0.01)$. B. Kaplan-Meier Analysis of 3-year DFS by Time from Diagnosis to HSCT. 3-year DFS was 71\% (95\% CI 44-87\%) in patients who had a diagnosis to HSCT time of <140 days and $26 \%$ (95\% CI 10-47\%) in those with diagnosis to HSCT time of $\geq 140$ days $(\mathrm{p}<0.01)$. C. Kaplan-Meier Analysis of 3year DFS in Patients who did not receive pre HSCT Chemotherapy and who were transplanted <140 days from Diagnosis. 3-year DFS was 80\% (95\% CI 51-93\%) in the 16 patients who did not receive pre HSCT chemotherapy and were transplanted $<140$ days from diagnosis. 
Table I

Patient Characteristics

\begin{tabular}{|c|c|}
\hline Characteristic & $\mathbf{N}(\%)$ \\
\hline Total patients & 37 \\
\hline \multicolumn{2}{|l|}{ Gender } \\
\hline Male & $18(49)$ \\
\hline Female & $19(51)$ \\
\hline Median age at diagnosis (years) & $11(1-21)$ \\
\hline \multicolumn{2}{|l|}{ Type of MDS } \\
\hline Primary & $20(54)$ \\
\hline Secondary & $17(46)$ \\
\hline \multicolumn{2}{|l|}{ Modified WHO MDS Classification (at diagnosis) } \\
\hline $\mathrm{RC}$ & $30(81)$ \\
\hline RAEB & $7(19)$ \\
\hline \multicolumn{2}{|l|}{ IPSS risk } \\
\hline Low & $1(3)$ \\
\hline INT-1 & $15(41)$ \\
\hline INT-2 & $21(57)$ \\
\hline \multicolumn{2}{|l|}{ Cytogenetics * } \\
\hline Monosomy 7 & $21(57)$ \\
\hline Trisomy 8 & $7(19)$ \\
\hline Normal/ Other ${ }^{* *}$ & $8(22)$ \\
\hline \multicolumn{2}{|l|}{ Pre HSCT chemotherapy } \\
\hline Yes & $7(19)$ \\
\hline No & $30(81)$ \\
\hline \multicolumn{2}{|l|}{ Time from diagnosis to HSCT (days) } \\
\hline$<140$ & $18(49)$ \\
\hline$\geq 140$ & $19(51)$ \\
\hline \multicolumn{2}{|l|}{ Year of HSCT } \\
\hline 1990-1999 & $24(65)$ \\
\hline 2000-2010 & $13(35)$ \\
\hline \multicolumn{2}{|l|}{ Pre-HSCT Blast count } \\
\hline$<5 \%$ & $27(73)$ \\
\hline $25 \%$ & $10(27)$ \\
\hline \multicolumn{2}{|l|}{ Graft source } \\
\hline UCB & $9(24)$ \\
\hline
\end{tabular}

Pediatr Blood Cancer. Author manuscript; available in PMC 2014 April 01. 


\begin{tabular}{|l|l|}
\hline Characteristic & N (\%) \\
\hline MRD & $15(41)$ \\
MURD & $7(19)$ \\
MMURD & $6(16)$ \\
\hline HLA match & \\
Match & $20(54)$ \\
Mismatch & $17(46)$ \\
\hline CsA containting GVHD prophylaxis & \\
Yes & $29(78)$ \\
No & $8(22)$ \\
\hline
\end{tabular}

MDS= Myelodysplastic Syndrome. Primary MDS= de novo. Secondary MDS $=4$ treatment-related, 8 with preceding idiopathic aplastic anemia, 3 with Schwachman Diamond syndrome, and 2 familial. WHO= World Health Organization. RC $=$ refractory cytopenia, RAEB= refractory anemia with excess blasts. IPSS $=$ international prognostic scoring system. INT- $1=$ intermediate 1. INT $-2=$ intermediate 2 . HSCT $=$ hematopoietic stem cell transplant. $\mathrm{UCB}=$ umbilical cord blood, $\mathrm{MRD}=$ matched related donor, $\mathrm{MURD}=$ matched unrelated donor, $\mathrm{MMURD}=$ mismatched unrelated donor. $\mathrm{CsA}=$ cyclosporine $\mathrm{A}$. GVHD= Graft versus host disease.

Cytogenetics not available for 1 patient.

*** Other chromosome abnormalities included: chromosome 3 abnormalities $(\mathrm{n}=1)$, isochromosome 7q $(\mathrm{n}=2), 11 \mathrm{q} 23$ abnormality $(\mathrm{n}=1)$, loss of $5 \mathrm{q}$ and $7 \mathrm{q}(\mathrm{n}=1)$. 
Table II

Multivanate Analysis of Overall Survival and Disease-Free Survival.

\begin{tabular}{|c|c|c|c|c|}
\hline & & Hazard Ratio & $95 \% \mathrm{CI}$ & P-value \\
\hline \multicolumn{5}{|l|}{3 Year Overall Survival } \\
\hline \multirow[t]{2}{*}{ Type of MDS } & Primary & 1.0 & & 0.23 \\
\hline & Secondary & 1.95 & $(0.65-5.83)$ & \\
\hline \multirow[t]{2}{*}{ Year of HSCT } & $<2000$ & 1.0 & & 0.02 \\
\hline & $\geq 2000$ & 0.17 & $(0.04-0.78)$ & \\
\hline \multirow[t]{2}{*}{ Pre-HSCT therapy } & Yes & 1.0 & & 0.02 \\
\hline & No & 0.29 & $(0.10-0.84)$ & \\
\hline \multirow[t]{2}{*}{ Gender } & Male & 1.0 & & 0.10 \\
\hline & Female & 2.49 & $(0.83-7.49)$ & \\
\hline \multicolumn{5}{|l|}{3 Year Disease-Free Survival } \\
\hline \multirow[t]{2}{*}{ Type of MDS } & Primary & 1.0 & & 0.33 \\
\hline & Secondary & 0.59 & $(0.21-1.71)$ & \\
\hline \multirow[t]{2}{*}{ Year of HSCT } & $<2000$ & 1.0 & & 0.07 \\
\hline & $\geq 2000$ & 0.3 & $(0.08-1.09)$ & \\
\hline \multirow[t]{2}{*}{ Pre-HSCT therapy } & Yes & 1.0 & & 0.03 \\
\hline & No & 0.3 & $(0.10-0.88)$ & \\
\hline \multirow[t]{2}{*}{ IPSS } & Int-1 & 1.0 & & 0.06 \\
\hline & Int-2 & 2.97 & $(0.95-9.33)$ & \\
\hline \multirow[t]{2}{*}{ Time from Diagnosis to HSCT } & $\geq 140$ days & 1.0 & & 0.02 \\
\hline & $<140$ days & 0.27 & $(0.09-0.80)$ & \\
\hline
\end{tabular}

IPSS= International prognostic score. MDS= Myelodysplastic syndrome. $\mathrm{UCB}=$ Umbilical cord blood, $\mathrm{MRD}=$ matched related donor, $\mathrm{MURD}=$ matched unrelated donor, MMURD= mismatched unrelated donor. 